\title{
HONGOS QUERATINOFILICOS OPORTUNISTAS EN EL PELAJE DE GATOS DOMESTICOS (Felix domesticus) EN LA CIUDAD DE VALPARAÍSO
}

\author{
(Opportunistic keratinophilic fungi in the fur of \\ domestic cats (Felix domesticus) in the city of Valparaiso)
}

\author{
J. P. Gallardo S. \& *E. Piontelli L. \\ Universidad Santo Tomás, Escuela de Medicina Veterinaria, Viña del Mar \\ *Universidad de Valparaíso, escuela de Medicina, Cátedra de Micología, Casilla 92 V, \\ Valparaíso, Chile (eduardo.piontelli@uv.cl)
}

Palabras clave: Hongos queratinofílicos, pelaje de gatosdomésticos, ciudad de Valparaíso

Key words: Keratinophilic fungi, fur of domestic cat, Valparaiso city

\section{RESUMEN}

En la ciudad de Valparaíso, se analizó mediante la técnica del tapete, la presencia de hongos queratinofílicos oportunistas en el pelaje de 198 gatos domésticos apa-rentemente sanos (96 mascotas y 102 de vida libre). Las siembras se efectuaron en duplicado en placas de Petri con agar Sabouraud glucosado incubándose en oscuridad a 35 C durante 15 días.

Se consideraron las variables, estilo de vida, edad, tipo de pelaje, sexo y época estacional, de las cuales sólo las dos primeras presentaron diferencias significativas en los taxa aislados. Un total de 130 individuos (65.6\%) fueron positivos a hongos filamentosos; 77 (59.2\%) correspondieron a gatos de vida libre y 53 (40.8\%) a gatos mascota.

Se obtuvieron 372 aislados fúngicos, de los cuales 335 (90.1\%) se consideraron como oportunistas, distribuidos en 22 géneros y 23 especies. De estos, 287 (77.1\%) se presentaron en gatos de vida libre y 85 (22.8 $\%)$ en mascotas. Los géneros dominantes fueron Aspergillus (53.5\%), Scopulariopsis (19.9\%), Fusarium (4,6\%) y el complex Alternaria alternata (3,8\%). Aspergillus, fue representado por 10 taxa, destacando A. flavus (15,9\%) y A. niger var. niger (13.4\%) y con menores frecuencias A.terreus, A. fumigatus, A.niger var. phoenicis y Emeri-cella nidulans (A. nidulans).

A. flavus y A. niger var. niger, se aislaron mayoritariamente en gatos de vida libre y lo mismo

Recibido el 5 de Junio 2007

Aceptado el 22 de Octubre 2007 sucede con prácticamente todas las especies del género. Scopulariopsis presentó solo 2 especies S.brevicaulis y S. candida. La primera fue la especie con mayor número de aislamientos (18\%) y la de mayor presencia en gatos de vida libre (77.9\%), mientras la segunda fue esporádica (1,6\%). Debe destacarse, por su rareza, que una cepa de $\mathbf{S}$. brevicaulis creció en cultivos asociada a su teleomorfo Microascus brevicaulis.

Los Onygenales fueron escasamente representados por Gymnashella citrina y Chrysosporium keratinophylum. Ningún dermatofito, incluyendo Microsporum canis, se aisló de los gatos analizados.

\section{ABSTRACT}

In the city of Valparaíso the presence of opportunistic fungi in the fur of 198 apparently healthy domestic cats (96 pets and 102 wild cats) was analyzed by means of the mat technique. Cultures were prepared in duplicate on Petri plates using glucosate Sabouraud agar and were incubated at 35C in the dark for 15 days. Factors such as variables, style of life, age, kind of fur, sex and seasonal period were considered, after which only the first two factors exhibited significant differences in the isolated taxa. A total of 130 specimen $(65,6 \%)$ resulted positive to filamentous fungi; being 77 (59,2\%) wild cats and $53(40,8 \%)$ pet cats.

Three hundred and seventy two fungal isolates were obtained, 335 of which $(90,1 \%)$ were considered as opportunistic, distributed in 22 genera and 23 species. Among these, $287(77,1 \%)$ were detected in wild cats and $85(22,8 \%)$ in pets. Dominant genera were 
Aspergillus (53,5\%), Scopulariopsis (19,9\%), Fusarium (4,6\& and the complex Alternaria alternata $(3,8 \%)$ : Aspergillus was represented by 10 taxa, A.flavus $(15,9 \%)$ and A. niger var. niger $(13,4 \%)$ exhibiting the highest frequency whereas A.terreus, A. fumigatus, A.niger var. phoenicis and Emericella nidulans (A. nidulans) showed the least occurrence.

A.flavus and A.niger varniger were mostly isolated from wild cats and this is true in the case of all species of the genus. Scopulariopsis revealed only two species, S.brevicaulis and S.candida. The former was the species with the highest number of isolations (18\%) together with the highest occurrence in wild cats $(77,9 \%)$, while the latter was sporadic. (1,6\%). Now then, it must be pointed out, due to its rareness, that a strain of S.brevicaulis grew in cultures associated to its teleomorph Microascus brevicaulis.

Onygenales were scarcely represented by Gymnashella citrina and Chrysosporium keratinophylum. None dermatophyte, Microsporum canis included, was isolated from the cats analyzed.

\section{INTRODUCCION}

El análisis de presencia y frecuencia de hongos queratinofílicos y queratinolíticos en el pelo de animales de compañía, junto con incrementar el conocimiento de su distribución, permite determinar sus propiedades oportunistas frente a factores adversos en un hospedador con bajas defensas y sus posibles implicaciones en salud pública debido a la convivencia con estos animales.

Por sus hábitos de vida, la piel y el pelo de los gatos y perros domésticos presentan e gran cantidad de propágulos fúngicos comunes en el suelo en diferentes zonas geográficas, tales como: Aspergillus, Penicillium, Cladosporium, Alternaria, Fusarium, Geotrichum, Curvularia, Paecilomyces, Rhizopus, Chrysosporium, Scopulariopsis, entre otros, (Piontelli \& Toro, 1987; Gambale et al, 1993; Rotstein et al., 1999; Cavalcanti et al., 2003). Entre las especies fúngicas queratinofílicas aisladas del pelaje de los animales de compañía y en hospitales veterinarios de Santiago, consideradas como potencialmente patógenas u oportunistas, puede apreciarse en Chile una dominancia de los géneros: Alternaria, Aspergillus, Cladosporium, Penicillium, Mucor, Scopulariopsis, sin considerar las levaduras (Flores et al., 1954; Jiménez ,1980; Piontelli \& Toro, 1987; Saldías, 1995; Perez, 2005).

Los hongos queratinofílicos son significativamente más frecuentes en gatos que en perros, sin embargo, estos últimos, presentan una mayor variedad debido a su mayor superficie de pelaje y por ende a su mayor contacto con el suelo (Piontelli \& Toro, 1987).
La mayoría de las enfermedades micóticas frecuentes de la piel de gatos son producidas por los dermatofitos: Microsporum canis, M.gypseum y Trichophyton mentagrophytes (Scott et al., 2001), siendo los principales representantes fúngicos degradadores de la queratina (Zaror et al., 1986).

En casi todo el mundo, el gato se considera como el principal reservorio de M.canis, representando un $90 \%$ de los dermatofitos aislados; los restantes suelen ser $\boldsymbol{T}$. mentagrophytes y M. gypseum, aunque con frecuencias variables en el perro (Sparkes et al., 1993; Cabañes, 2000; Thomsom, 2002).

En nuestro país los estudios realizados por Reyes (1998) y Corfio (1998) en caninos, Andreu ( 2000) en conejos, hamsters y ratones, y Perez (2005) en hospitales veterinarios de Santiago, determinaron el marcado predominio de T. mentagrophytes en muestras obtenidas desde animales de compañía infiriendo que la contaminación ambiental directa e indirecta por dermatofitos guarda una estrecha relación con la portación de hongos en animales domésticos.

Si bien es cierto que los dermatofitos producen las micosis más comunes en los animales de compañía, en presencia de factores predisponentes que disminuyen su inmunidad, algunos hongos considerados saprofíticos pueden asumir roles patogénicos oportunistas e invadir los tejidos del estrato córneo favorecidos por una temperatura menor a los $37^{\circ} \mathrm{C}$ y la presencia de hierro y nutrientes básicos en la grasa y la transpiración ( Scott et al., 2001; Paixao et al., 2001). Para diferenciar entre un hongo patógeno y un contaminante, es importante considerar el número y de veces que se aísla el agente, identificar la fuente de infección y especialmente la presencia de elementos fúngicos en el estrato córneo.

La diferencia entre hongos queratinolíticos y queratinofílicos puede ser más cuantitativa que cualitativa; ambos pueden producir diversas enzimas degradadoras de queratina (proteinasas extracelulares como colagenasa, elastasa y lipasas que facilitan la penetración de la película lipídica de la epidermis pero los queratinolíticos producen grandes cantidades de éstas en especial proteasas (queratinasas) (Kunert, 2000; Sharma \&Rajak, 2003).

La finalidad de nuestra investigación fue aislar e identificar, en la ciudad de Valparaíso, los hongos filamentosos queratinofílicos o queratinolíticos oportunistas presentes en el pelaje de gatos domésticos, aparentemente sanos (dermatológicamente) y correla-cionar su presencia con las variantes edad, estilo de vida y tipo de pelaje.

\section{MATERIALES Y METODOS}

Se tomaron muestras en duplicado del pelaje de 198 gatos domésticos, 96 mascotas y 102 de vida libre aparente- 
mente sanos, sin signos de patologías dermatológicas, a contar del mes de junio de 2005 hasta agosto de 2006, en la ciudad de Valparaíso. Estas muestras se analizaron en el Laboratorio de Micología de la Facultad de Medicina de la Universidad de Valparaíso.

\section{Obtención de las muestras}

Las muestras fueron obtenidas frotando varias veces un trozo de alfombra estéril de $5 \times 5 \mathrm{~cm}$ (transportada dentro de una placa de Petri estéril), sobre la superficie del manto piloso en sentido inverso a la orientación del pelaje, desde zonas de la cabeza, cuello patas y pecho de los animales utilizando el método de Mariat \& AdamCampos (1967). Posteriormente se introdujo nuevamente el trozo de alfombra en la placa de Petri que lo contenía sellándola con Alusa Plast. Las muestras se transportaron al laboratorio para su procesamiento en un plazo no superior a las 48 horas, período en el cual se mantuvieron refrigeradas a $4^{\circ} \mathrm{C}$.

\section{Criterios de inclusión.}

Se estudiaron gatos domésticos de vida libre o mascotas, sin antecedentes dermatológicos, de cualquier raza o mestizaje, de ambos sexos, mayores de un mes y sin tratamiento antifúngico por lo menos 30 días antes de la obtención de la muestra (sólo en las mascotas domésticas).

La condición considerada como «dermatológicamente sano», se evaluó en un examen clínico descartándose todos aquellos animales que presentaron lesiones dermatológicas diversas (en ambos grupos analizados).

Se considera como gato mascota o de vida interior a aquellos que permanecen la mayor parte de su tiempo dentro de la casa o bien tiene una salida al exterior escasa $\mathrm{y}$ por gato de vida libre a aquellos que permanecen poco o ningún tiempo dentro de las casas o en distintos inmuebles (Bodegas, Galpones, Negocios) pero mayoritariamente pasan largas horas, especialmente de noche, en el exterior explorando y defendiendo su territorio. Lógicamente estos gatos tienen contacto con otros gatos, cazan pájaros, roedores, comen insectos y están expuestos a otros peligros como atropellos, intoxicaciones, ataques de perros y otros accidentes.

\section{Edad y tipo de pelaje}

Los animales fueron seleccionados de acuerdo a dos categorías etarias, hasta un año y mayores de 2 años. Se muestrearon animales de pelo corto y de pelo largo, determinación que se efectuó en el momento del examen clínico

\section{Métodode siembra}

La siembra se efectuó en duplicado en placas de Petri con agar Sabouraud glucosado al $2 \%$ adicionado de cloramfenicol a razón de 0.25 gramos por litro, mediante la impresión repetida ( 2 a 3 veces) de la superficie usada de la alfombra sobre toda la superficie del agar para depositar las escamas y los pelos retenidos en ella. Las placas sembradas se incubaron en oscuridad a $35+/-1^{\circ} \mathrm{C}$ en condiciones de aerobiosis, realizándose la observación microscópica entre la primera y la segunda semana. Los cultivos positivos se mantuvieron en observación por un período de 30 días

\section{Identificación microscópica de los hongos}

A partir de las colonias, se realizó una preparación microscópica con Lactofenol azul de algodón con el objetivo de visualizar las estructuras de fructificación. Luego fueron resembrados en PDA o en medios especiales de identificación según el género y lo indicado en las monografías correspondientes hasta el nivel de especie (Principalmente: Domsch et al., 1980; Currah, 1985; Samson et al., 2000; De Hoog et al., 2000, Klich, 2002).

\section{Análisis estadístico}

Se aplicó el coeficiente de correlación de Spearman (variables cualitativas) para relacionar la frecuencia de presentación de las diferentes especies de hongos queratinofílicos oportunistas, con las condiciones género, estilo de vida, edad, tipo de pelaje y entre gatos de vida libre y mascotas. Se aplicó el test de diferencias de proporciones, para comparar las condiciones género, estilo de vida, edad y tipo de pelaje para cada una de las especies de hongos queratinofilícos potencialmente patógenos que tuvieron una frecuencia igual o mayor a 7 gatos.

\section{RESULTADOS}

Durante un período anual, se estudiaron un total de 198 gatos sin lesiones macroscópicas aparentes en su pelaje, de ellos 130 individuos $(65.65 \%)$ fueron positivos a hongos filamentosos; $77(59.23 \%)$ correspondieron a gatos de vida libre y $53(40.76 \%)$ a gatos mascota, mientras $68(34.34 \%)$ no presentaron ningún tipo de hongos. De los gatos positivos, $124(95.38 \%)$ presentaron cepas potencialmente patógenas u oportunistas (crecimiento a $35-36^{\circ} \mathrm{C}$ ) y el $28.46 \%$ de éstos $(37)$ se asociaron además a otras cepas fúngicas saprofíticas. Sólo 6 de los 130 gatos positivos $(4.61 \%)$ presentaron exclusivamente cepas consideradas saprofíticas.

Se obtuvieron 372 aislamientos fúngicos, de los cuales $335(90.05 \%)$ se consideraron como oportunistas, distribuidos en 22 géneros, identificándose 23 especies. Del total de aislamientos $287(77.15 \%)$ correspondieron a gatos de vida libre y $85(22.84 \%)$ a gatos mascotas.

Entre los taxa considerados oportunistas, puede apreciarse una franca dominancia de los integrantes de los géneros; Aspergillus 199 (53.49\%), Scopulariopsis 74 
Tabla 1. Hongos queratinofilicos potencialmente patógenos y saprófitos aislados de gatos de vida libre y mascotas de la ciudad de Valparaíso

\begin{tabular}{|c|c|c|c|c|c|c|c|c|c|c|c|c|}
\hline \multirow[t]{2}{*}{ Taxa fúngicos } & \multicolumn{2}{|c|}{ Sexo } & \multicolumn{2}{|c|}{ Tipo vida } & \multicolumn{2}{|c|}{ Edad } & \multicolumn{2}{|c|}{ Pelo } & \multicolumn{2}{|c|}{ Estación } & \multirow[t]{2}{*}{ Tot. } & \multirow[t]{2}{*}{$\%$} \\
\hline & $\mathbf{H}$ & $\mathbf{M}$ & Masc. & V.li. & $<1$ & $>2$ & \begin{tabular}{|l|l|} 
P.C \\
\end{tabular} & $\overline{\text { P.L }}$ & $\mathbf{O} / \mathbf{I}$ & $\mathbf{P} / \mathbf{V}$ & & \\
\hline Alternaria alternata (Fr.) Keissler complex & 6 & 8 & 2 & 12 & 6 & 8 & 5 & 9 & 8 & 6 & 14 & 3,8 \\
\hline Aspergillus clavatus Dezm. & 2 & 2 & 0 & 4 & 4 & 0 & 3 & 1 & 2 & 2 & 4 & 1,1 \\
\hline Aspergillus flavus Link & 27 & 32 & 10 & 49 & 30 & 29 & 27 & 32 & 31 & 28 & 59 & $\overline{15,9}$ \\
\hline Aspergillus fumigatus Fres. & 10 & 12 & 5 & 17 & 12 & 10 & 12 & 10 & 14 & 8 & 22 & 5,9 \\
\hline Aspergillus niger var. phoenicis Corda & 7 & 8 & 1 & 14 & 7 & 8 & 7 & 8 & 10 & 5 & 15 & 4,0 \\
\hline Aspergillus niger var. niger Van Tieghem & 25 & 25 & 6 & 44 & 19 & 31 & 25 & 25 & 31 & 19 & 50 & 13,4 \\
\hline Aspergillus parasiticus Speare & 3 & 2 & 0 & 5 & 1 & 4 & 4 & 1 & 4 & 1 & 5 & 1,3 \\
\hline Aspergillus sidowi (Bain\&Sort) & 1 & 0 & 1 & 0 & 1 & 0 & 1 & 0 & 1 & 0 & 1 & 0,3 \\
\hline Aspergillus terreus Thom & 10 & 13 & 2 & 21 & 12 & 11 & 12 & 11 & 15 & 8 & 23 & 6,2 \\
\hline Aspergillus ustus (Bainier)Tom \& Church & 3 & 3 & 2 & 4 & 3 & 3 & 4 & 2 & 3 & 3 & 6 & $\overline{1,6}$ \\
\hline Aureobasidium pullulans (De Bary) Arnaud & 3 & 4 & 4 & 3 & 1 & 6 & 5 & 2 & 3 & 4 & 7 & $\overline{1,9}$ \\
\hline Botryotrichum piluliferum Sacc.\&March1 & 0 & 1 & 1 & 0 & 0 & 1 & 1 & 0 & 0 & 1 & 1 & 0,3 \\
\hline Candida sp. & 0 & 1 & 1 & 0 & 1 & 0 & 1 & 0 & 0 & 1 & 1 & 0,3 \\
\hline Chaetomium spp. & 3 & 0 & 0 & 3 & 0 & 3 & 2 & 1 & 1 & 2 & 3 & $\overline{0,8}$ \\
\hline Chrysosporium keratinophylum (Frey) Charmich. & 1 & 2 & 0 & 3 & 2 & 1 & 2 & 1 & 3 & 0 & 3 & 0,8 \\
\hline Emericella nidulans (Eidam) Bullí. & 6 & 8 & 1 & 13 & 7 & 7 & 8 & 6 & 6 & 8 & 14 & 3,8 \\
\hline Fusarium spp. & 10 & 7 & 4 & 13 & 7 & 10 & 7 & 10 & 9 & 8 & 17 & 4,6 \\
\hline Gymnaschella citrina Siegler \& Currah & 1 & 3 & 2 & 2 & 0 & 4 & 1 & 3 & 3 & 1 & 4 & 1,1 \\
\hline Microascus brevicaulis Abbot et al. & & 1 & & 1 & & 1 & & 1 & & 1 & 1 & 0,3 \\
\hline Microascus trigonosporus Emmo. \& Dodge & 0 & 1 & 0 & 1 & 0 & 1 & 0 & 1 & 1 & 0 & 1 & 0,3 \\
\hline Mucor sp. & 6 & 10 & 12 & 4 & 8 & 8 & 10 & 6 & 8 & 8 & 16 & 4,3 \\
\hline Neocosmospora vasinfecta E.F. Smith & & 2 & & 2 & & 2 & & 2 & 2 & & 2 & 0,5 \\
\hline Paecilomyces variotii Bain & 4 & 3 & 2 & 5 & 4 & 3 & 4 & 3 & 3 & 4 & 7 & 1,9 \\
\hline Papulospora $\mathrm{sp}$. & 2 & 1 & 2 & 1 & 2 & 1 & 2 & 1 & 1 & 2 & 3 & 0,8 \\
\hline Penicillium spp. & 0 & 3 & 3 & 0 & 2 & 1 & 2 & 1 & 2 & 1 & 3 & 0,8 \\
\hline Rhizopus oryzae Went \& Prinsen Geerlings & 0 & 1 & 0 & 1 & 1 & 0 & 1 & 0 & 0 & 1 & 1 & 0,3 \\
\hline Rhodotorula spp. & 1 & 4 & 4 & 1 & 1 & 4 & 1 & 4 & 1 & 4 & 5 & 1,3 \\
\hline Scedosporium opiospermum (Sacc.) Sacc. & 2 & 2 & 1 & 3 & 3 & 1 & 3 & 1 & 2 & 2 & 4 & 1,1 \\
\hline Scopulariopsis brevicaulis Bain. & 30 & 37 & 15 & 52 & 39 & 28 & 32 & 35 & 42 & 25 & 67 & 18,0 \\
\hline Scopulariopsis candida (Gueguen) Vuill. & 4 & 2 & 1 & 5 & 3 & 3 & 5 & 1 & 3 & 3 & 6 & 1,6 \\
\hline Sepedonium sp. & 2 & 3 & 3 & 2 & 2 & 3 & 2 & 3 & 2 & 3 & 5 & 1,3 \\
\hline Trichoderma sp. & 1 & 0 & 0 & 1 & 0 & 1 & 1 & 0 & 0 & 1 & 1 & 0,3 \\
\hline Ulocladium sp. & 1 & 0 & 0 & 1 & 0 & 1 & 0 & 1 & 0 & 1 & 1 & 0,3 \\
\hline Totales: & 172 & 200 & 85 & 287 & 179 & 193 & 191 & 181 & 210 & 162 & 372 & 100 \\
\hline
\end{tabular}

(19.89\%), Fusarium 19 (4,6\%) y del complex Alternaria alternata 14 (3.8\%) (Tabla 1, Fig.1-2).

El género con mayor número de especies fue Aspergillus, representado por 10 taxa (Fig.3). Las especies dominantes más frecuentes fueron: A. flavus con 59 aislamientos (15,9\%), y $\boldsymbol{A}$. niger var. niger con 50 aislamientos (13.4\%), le siguen con menores frecuencias A. terreus, A. fumigatus, A.niger var. phoenicis y Emericella nidulans (A. nidulans) (Tabla 1).

A. flavus (49/10) y A. niger var. niger (14/1), se aislaron mayoritariamente en gatos de vida libre y lo mismo sucede con prácticamente todas las especies del género aisladas (Tabla 1).

Scopulariopsis fue el segundo género en importancia con solo 2 especies S.brevicaulis y $\boldsymbol{S}$. candida. $\mathrm{La}$ primera obtuvo la mayor frecuencia de todos los taxa aislados con 67 aislamientos (18.0\%) y con una mayor proporción en gatos de vida libre $(77.9 \%)$. Una cepa de $S$. brevicaulis incluyó un raro aislamiento de su teleomorfo asociado Microascus brevicaulis. 


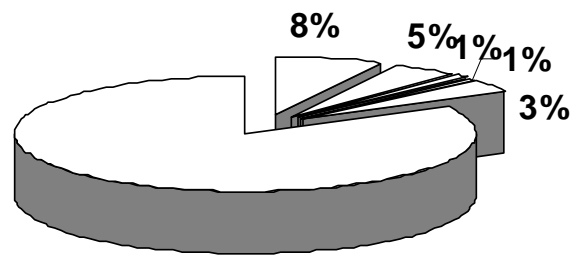

$82 \%$

$\square$ Aspergillus $8 \%$

$\square$ Fusarium $1 \%$

$\square$ Mucor spp. 3\%

$\square$ Scopulariopsis $5 \%$

$\square$ A. alternata $1 \%$

$\square$ Otros $82 \%$

Figura 1. Principales géneros queratinofílicos aislados en gatos mascotas

Los restantes taxa, fueron principalmente integrados por representantes del género Fusarium spp. $(4,6 \%)$, principalmente en los gatos de vida libre, Mucor spp. (4,3\%), principalmente en mascotas y Alternaria alteranata complex $(3,76 \%)$, principalmente en gatos de vida libre; las otras especie obtuvieron bajos porcentajes (Tabla 1, Fig.1-2). Los integrantes de los Onygenales fueron solamente representados por Gymnashella citrina y Chrysosporium keratinophylum con un 1,87\%.

Llama la atención que Microsporum canis, ni otro dermatofito se aisló en ningunos de los gatos analizados (Tabla 1).

Las especies que consideramos como saprófitas, fueron representadas por los géneros Mucor spp., Papulospora sp., Penicillium spp., Sepedonium sp.y Aureobasidium pullulans.

A pesar que este trabajo no incluía hongos leva-

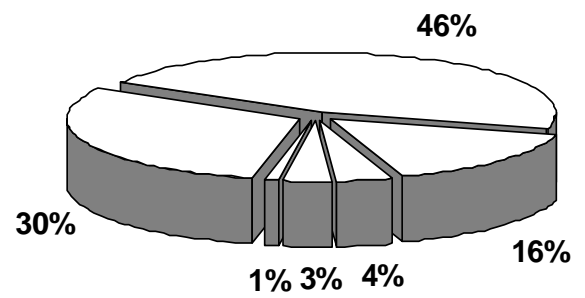

\section{$\square$ Aspergillus $46 \% \quad \square$ Scopulariopsis $16 \%$ \\ $\square$ Fusarium $4 \%$ \\ $\square$ Mucor spp. $1 \%$ \\ $\square$ A. alternata $3 \%$ \\ $\square$ Otros $30 \%$}

Figura 2. Principales géneros queratinofílicos aislados en gatos de vida libre

duriformes, destacamos la presencia de Rhodotorula spp en 5 gatos y de Candida sp. en 1 .

Respecto de la variable edad, no existieron diferencias significativas, salvo para la especie Scopulariopsis brevicaulis quien presentó una mayor proporción de portadores en el grupo de animales menores de un año ( $\mathrm{P}=0,05)$, a diferencia de Aspergillus niger, que presentó una mayor proporción de portadores en los animales mayores de 2 años $(\mathrm{P}=0,05)$.

En las variables sexo y tipo de pelo, no existió diferencia significativa para ninguna de las especies estudiadas.

En la estación fría (Otoño-invierno) se aislaron el 56\% de las especies. S.brevicaulis, A.niger var. niger, A.fumigatus y A.terreus fueron mayoritarios, con porcentajes entre el 62 al $65 \%$. Las cepas restantes no mostraron mayores cambios estacionales.

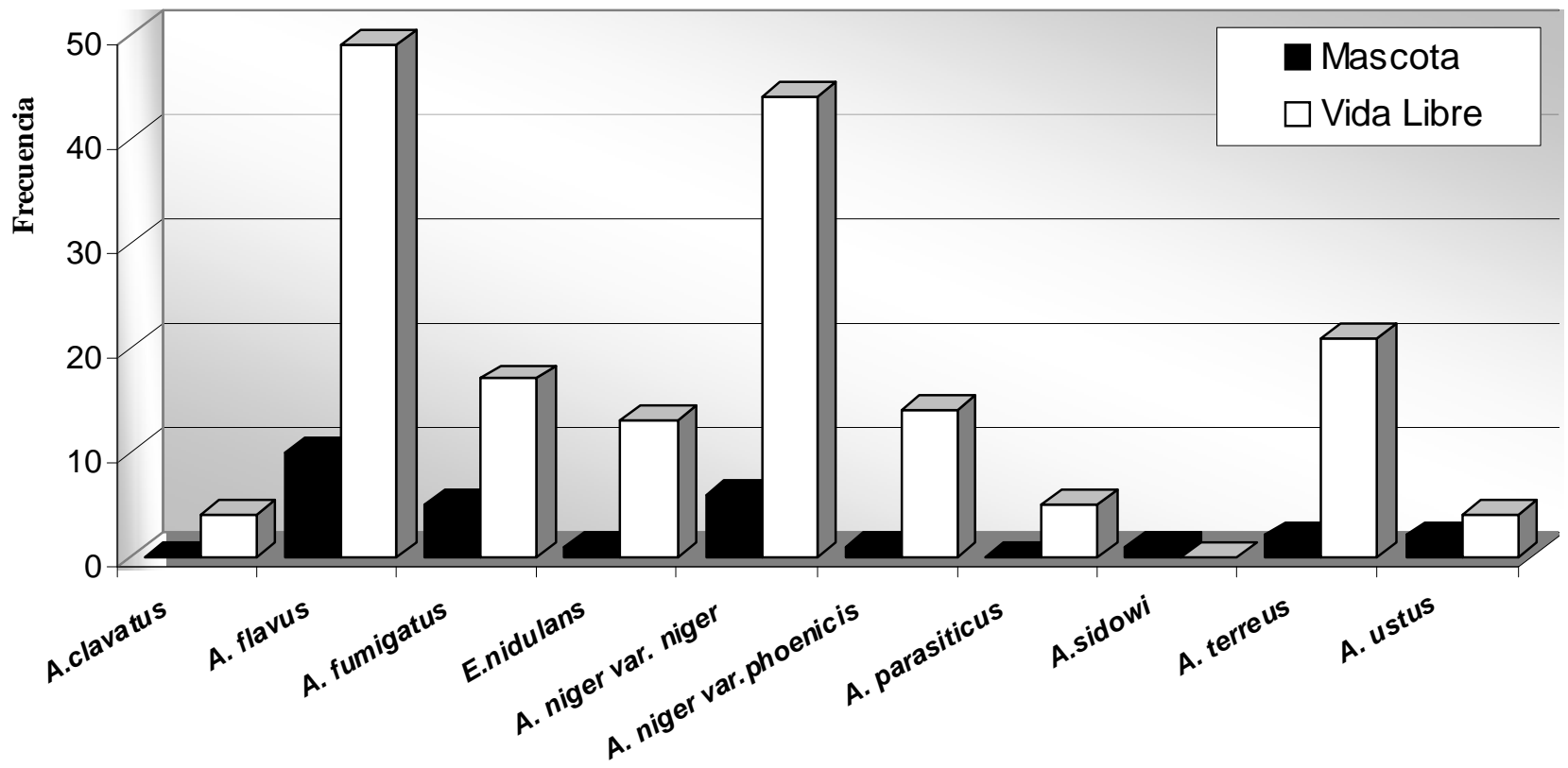

Figura 3. Especies del géneros Aspergillus aisladas de gatos mascota y vida libre 

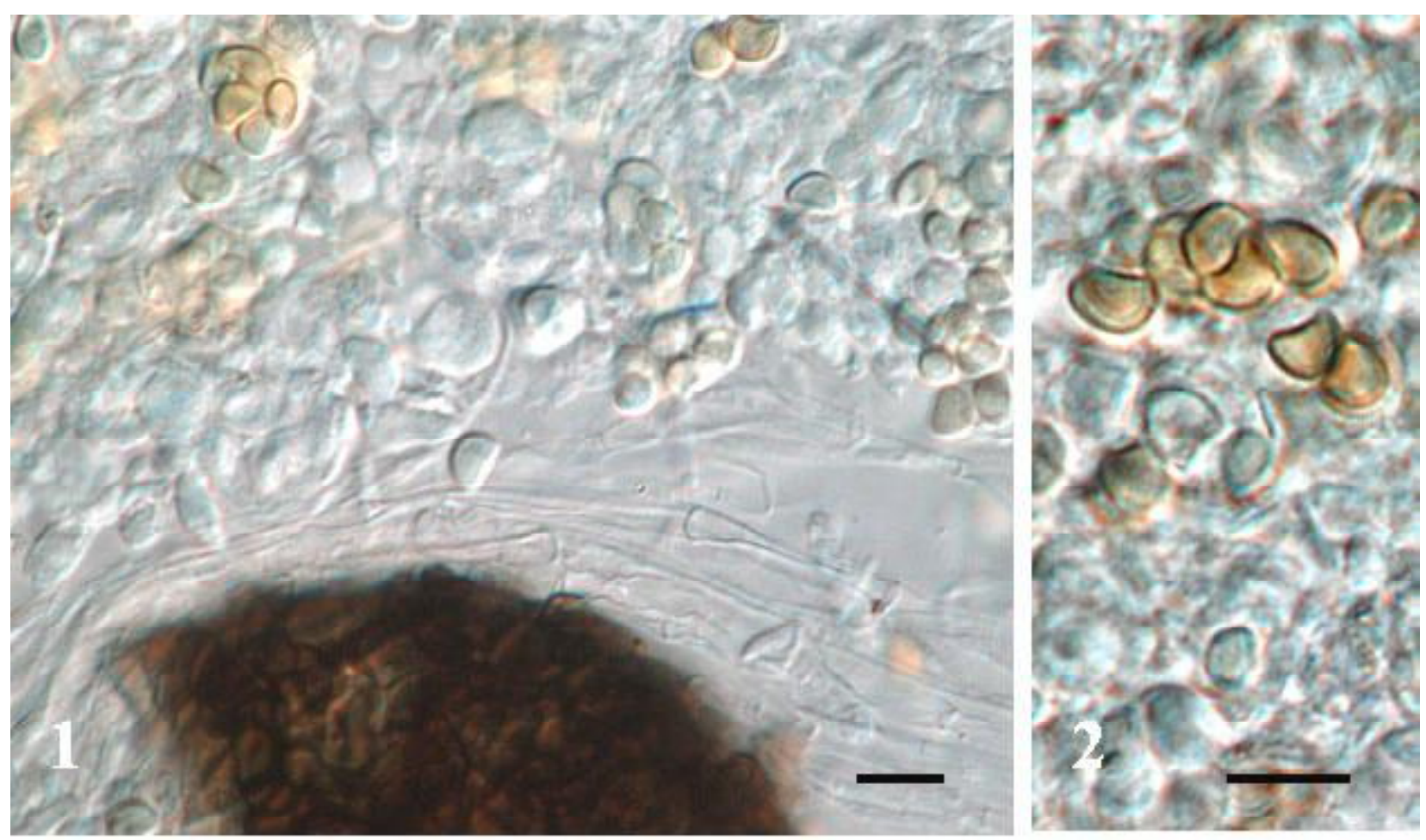

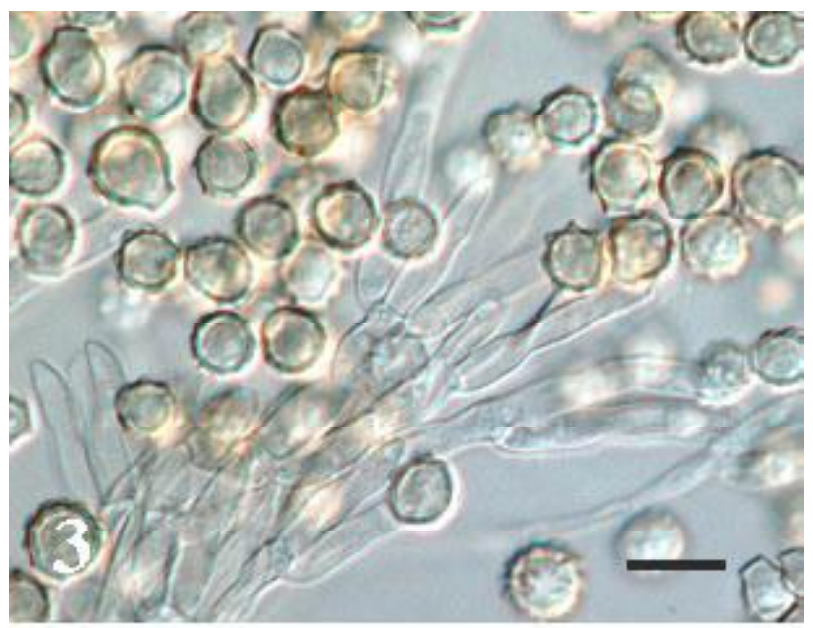

Figura 4. 4,1-4,2. Microascus brevicaulis, parte del peridio $\mathbf{y}$ ascosporas ; ascos $\mathbf{y}$ ascosporas a mayor aumento (X2000). 4,3. Scopulariopsis brevicaulis (anamorfo ).Conidióforos y conidios. Barra 10 um

\section{DISCUSION}

Existen pocos trabajos a nivel nacional de hongos queratinofílicos considerados oportunistas en gatos asintomáticos o aparentemente sanos. La gran mayoría de los autores enfoca su atención en las especies patógenas Piontelli \& Toro, 1987 (perros y gatos); Reyes, 1998 (perros); Corfío, 1998 (perros), Andreu, 2000 (conejos, hámsters y ratones) y Thomsom, 2002 (perros con lesiones), principalmente dermatofitos, clasificando al resto de los aislamientos fúngicos como contaminantes o saprófitos como los encontrados en los suelos de hospitales veterinarios, desestimando su oportunismo potencial (Jimenez, 1980; Saldías et al., 1995; Perez, 2005). Entre los géneros más frecuentes encontrados por estos últimos autores, destacan los integrantes de los géneros Aspergillus, Penicillium, Cladosporium, Alternaria, Scopulariopsis y levaduras, concordando en general, con los mayores aislamientos de la presente investigación (Aspergillus, Scopulariopsis y Alternaria). Los integrantes de Fusarium, principalmente $\boldsymbol{F}$. oxysporum y F. verticillioides (datos no incluidos en Tabla 1) que obtuvieron el tercer porcentaje de aislamiento, no se mencionan por los autores precedentes dentro de los más aislados.

Pudimos observar que los gatos de vida libre son significativamente más portadores de una micota fúngica filamentosa queratinofílica potencialmente patógena que los gatos mascotas de vida interior, para todas las especies fúngicas analizadas. Lo anterior puede asociarse por el mayor contacto de estos felinos con los diversos ambientes del suelo, a veces lejanos de su hábitat, por disputas territoriales con otros gatos o el contacto con sus presas (roedores, pájaros, reptiles e insectos).

Scopulariopsis brevicaulis, fue la especie oportunista más frecuente encontrada con mayor proporción de portadores en gatos de vida exterior y menores de un año. Su mayor o menor presencia en la microbiota 
de la piel, en comparación con adultos, no tiene una clara explicación, puede atribuirse a cambios bioquímicos de la piel, secreciones, reemplazo constante del pelo, mayor contacto con el suelo por continuos juegos y su mayor actividad motora propia de la edad. Sin embargo, debe considerarse que las especies de Scopulariopsis (en especial $\boldsymbol{S}$. brevicaulis) son de amplia distribución en los suelos, desde las zonas árticas a las desérticas, excrementos de herbívoros, alimentos ensilados, en detritus vegetales y en substratos queratínicos animales (Domsch et al., 1980). Sus conidios se dispersan por vía aérea y son aislados desde la atmósfera exterior con cierta constancia en bajas concentraciones (Airaudi \& Marchisio, 1996). La capacidad de S. brevicaulis de degradar un amplio rango de fuentes carbonadas tales como celulosa, aceites, fenoles, además de ser ser un débil queratinolítico con capacidad de crecer a $37^{\circ} \mathrm{C}$ ( Domsch et al., 1980 ; Filippello -Marchisio et al., 2000), lo convierte en una especie oportunista en animales y humanos debilitados o inmunodeprimidos de cualquier edad (de Hoog et al., 2000; Gugnani, 2000).

La presencia de esta especie junto a $\mathbf{S}$. candida, es indicativo de lo favorable que es este biotopo para la supervivencia y dispersión de sus conidios en el pelaje de los gatos.

S.brevicaulis, presenta siempre en cultivos su forma asexual y se consideraba incapaz de producir su forma sexuada, sin embargo, hace un decenio, efectuando cruzamientos con muchas cepas de esta especie, Abbot et al. (1998), pudieron obtener su forma sexual (Microascus brevicaulis), pero con muy pocas cepas con peritecios fértiles y solo con cepas provenientes de Alberta (Canadá). En el presente estudio, debemos destacar el raro hallazgo de una nueva cepa de M.brevicaulis (heterotálica) con abundante producción de peritecios fértiles y ascosporas reniformes (Fig.4) en adición a los típicos conidios anelídicos en cadena de su anamorfo. Es posible asociar este hallazgo a una nueva población sexual con restricción geográfica del hemisferio sur.

En nuestro país Piontelli \& Toro (1987), aislaron especies de Scopulariopsis en el (25.65\%) de los perros y (19.54\%) de los gatos en Valparaíso, siendo Scopulariopsis candida la especie más frecuente en ambos animales (12.04\% y 6.89\%), seguida por Scopulariopsis brevicaulis con $(6.28 \%$ y $5.74 \%)$ respectivamente. Con nuestra investigación, podemos suponer que en 20 años las poblaciones de S.brevicaulis en la misma zona geográfica podrían haber aumentado en los suelos, y la temperatura de cultivo un factor selectivo para su desarrollo.

En USA, Scopulariopsis es uno de los géneros comúnmente aislados desde gatos sanos, felinos silvestres y perros, considerándose como un género habitual en la microbiota de la piel de estos animales (Moriello \& De
Boer, 1991; Rotstein et al., 1999; Boyanowski et al., 2000).

En Brasil, diferentes estudios en gatos domésticos y otros felinos salvajes y silvestres en cautiverio, encontraron este género entre los más frecuentes, en la piel de estos animales (Gambale et al., 1993; Cavalcanti et al., 2003; Levi et al., 2006).

En Francia, Sierra et al. (2000), encuentra a integrantes del género Scopulariopsis en el (41.8\%) de gatos seropositivos de FIV y FeLV y en España, Cabañez et al. (1996), en el $(65.7 \%)$ de los perros en estudio, con una mayor frecuencia en verano y en otoño, no observando diferencias estadísticamente significativas en la microbiota con respecto edad, género, largo del pelo, mestizaje o raza.

Caretta et al. (1989), lo mencionan como el género más frecuente después de Alternaria en perros y gatos en Italia; mientras en Finlandia, es el quinto género más común en animales domésticos y de laboratorio, con sospecha de dermatofitosis (Aho, 1983). Los integrantes de este género no solo se han detectado en gatos y perros sino en otros mamíferos, animales de granja y aves (Bagy, 1986; Ali-Shtayeh et al., 1988; Camin et al., 1998). Losintegrantes del género Scopulariopsis, muestran afinidad especial por las uñas, causando frecuentes onicomicosis resistentes a la mayoría de los antimicóticos (Aho, 1983; Cuenca-Estrella, et al., 2006).

En medicina humana $S$. brevicaulis y otras especies del género son patógenos emergentes ya sea en micosis superficiales y profundas en pacientes con factores predisponentes y en especial en SIDA (Migrino et al., 1995; Schinabeck \& Ghannoum, 2003; Romano et al., 2005).

Los aislamientos de Aspergillus, abarcaron la mayoría de los cepas fúngicas, siendo A. flavus, A. niger var. niger, A terreus y $A$. fumigatus las más frecuentes en especial en los gatos de vida libre. Los integrantes del género son comunes en ambientes tropicales y cálidos más que en los temperados y fríos, en especial sobre substratos vegetales, suelo, alimentos, granos, especies y en el aire de ambientes externos e internos (Samson et al., 2000). Su presencia en el pelaje de animales domésticos es variable según la localización geográfica, como se demuestra en estudios de la microbiota de la piel de gatos y perros, felinos salvajes, silvestres y en cautiverio en Brasil, determinando que este género es uno de los más prevalentes (sin mencionar las especies), según los tipos de ambiente y tipos de muestreo efectuados (Gambale $e t$ al., 1993; Paixao et al., 200; Cavalcanti et al., 2003; ; Levi et al., 2006).

En USA y Europa, es uno de los más frecuentes en gatos domésticos mascotas y felinos silvestres (Cabañes et al.,1996; Caretta et al., 1989; Moriello \& De Boer, 1991; Rotstein, et al., 1999; Sierra et al., 2000). En 
Jordania, las especies de Aspergillus ocuparon el segundo lugar en frecuencia tras Alternaria en el pelaje de los gatos, siendo A. niger, A. flavus y A. terreus los más frecuentes (Ali-Shayed et al., 1988), una situación muy similar a la ocurrida en la presente investigación. En Chile se han aislado en animales de compañía y en hospitales veterinarios (Jimenez, 1980; Saldías et al., 1995; Perez, 2005). Piontelli y Toro (1987), lo aislaron como patógeno oportunista en muy baja frecuencia desde perros y gatos en Valparaíso.Los integrantes de este género, en especial A. terreus y A. niger, pueden producir fatales micosis sintémicas en pastores alemanes (Mullaney et al., 1983; Kabay et al., 1985; Day et al., 1986) y localizarse en los senos nasales y paranasales en perros y gatos (Mortellaro et al, 1989; Greene, 1998), con escasos resultados favorables a la terapia. La identificación hasta nivel de especie de estos hongos es cada vez más importante, debido a que algunas presentan una mayor virulencia y una respuesta distinta a los antifúngicos.

El gran porcentaje de animales domésticos portadores de conidios de Aspergillus en su piel, considerados como saprófitos contaminantes, promueve su distribución y sobrevivencia y puede considerarse como otro posible reservorio de hongos oportunistas para el hombre.

Entre los remanentes hongos filamentosos queratinofílicos oportunistas comunes en el suelo, pero aislados en porcentajes inferiores al 5\%, debemos considerar a los integrantes del género Fusarium, (un 60\% de las cepas determinadas como F.oxyspoum y F.verticillioides; datos no incluidos en Tabla 1) fueron los terceros en frecuencia de presencia. Anteriormente en Chile sus integrantes fueron aislados en bajos porcentajes desde perros y gatos, con cifras cercanas al 1\% (Piontelli $\&$ Toro, 1987). En Brasil se presentan comúnmente desde el pelaje de felinos domésticos, silvestres y salvajes en cautiverio (Gambale et al., 1993; Paixao et al., 2001; Cavalcanti et al., 2003; Levi et al., 2006), como también en USA, (Moriello \& DeBoer, 1991; Rotstein et al,, 1999; Boyanowski et al., 2000). En España, Cabañes et al. (1996), aislaron Fusarium en baja frecuencia desde perros, señalando una mayor presencia en los meses de verano. En Jordania fue mayoritario entre los hongos del pelaje de gatos, siendo $\boldsymbol{F}$. verticillioides la especie dominante (AliShayed et al., 1988). El género Fusarium incluye más de 100 especies (Leslie \& Summerell, 2005), es un taxon filamentoso cosmopolita del suelo, que se asocia a muchos restos vegetales y cereales. Es común principalmente en ambientes cálidos y aún cuando es un gran patógeno vegetal es causante de diversas micosis opotunistas en el hombre y los animales y de la producción de varias importantes micotoxinas de interés en salud pública (De Hoog et al., 2000; Samson et al.; Kluger et al., 2004).
Los integrantes del complex Alternaria alternata, hongos dematiáceos cosmopolitas, colonizadores primarios de muchos substratos vegetales y por ende, comúnmente aislados desde el filoplano de las plantas, suelo, alimentos, semillas y el aire de ambientes internos y externos (Samson et al., 2000). Este género cuenta con alrededor de 50 especies, de las cuales el complex Alternaria alternata, es el más aislado desde pelo y piel de numerosas especies animales, quizás debido a su notable capacidad de proliferar en sustratos asociados a la queratina (Ali-Shtayeh, 1988), y su alta presencia en los suelos y vegetación.

En la presente investigación presentó una frecuencia menor a la descrita por Piontelli \& Toro (1987), con un $11.5 \%$, mientras en gatos menores de 1 año suele llegar del 30 al 50\% en otras investigaciones nacionales, por lo cual el aislamiento del complex A.alternata u otra especie desde perros con lesiones evidentes debe inducir a pensar en una acción patógena oportunista (Flores,1954; Jiménez, 1980; González, 1986), causante de onicomicosis, micosis ulcerativas cutáneas y queratitis en animales de compañía (Cabañes et al., 2000). La alta frecuencia de los integrantes del género Alternaria parece ser común en el pelaje y piel con o sin lesiones en gatos, perros, caballos, aves de corral, conejos, ovejas, cabras y otros mamíferos silvestres que se describen en la bibliografía analizada en varíos países (Aho, 1983; Ali-Shayed et al., 1988; Moriello \& DeBoer, 1991; Cabañes et al., 1996; Rotstein et al., 1999; Boyanowski et al., 2000).

Específicamente en el pelaje de los gatos, Caretta et al. (1989), lo aisla como el género más frecuente en el norte de Italia, una situación similar se observa en Europa Asia y Sud américa (Ali-Shayed et al., 1988; Gambale et al., 1993; Khosravi, 1996; Cavalcanti et al., 2003), siendo generalmente el complex A. alternata la especie más frecuente.

En humanos ha sido causante de infecciones víscerales y osteomielitis, onicomicosis, micosis oculares y cuadros alérgicos (De Hoog et al.,2000).

Debemos destacar también a pesar de su escasa presencia 2 especies cosmopolitas reportadas como agentes de distintas patologías en animales y humanos,tales como Paecilomyces varioti y Scedosporium apiospermum, que pueden obtenerse a partir de muestras procedentes del suelo, tierra de macetas, estíercol, restos vegetales y productos alimenticios (Elliot et al., 1984; 2000; De Hoog et al., 2000; San Juan et al., 2004).

No se detectó la presencia de dermatofitos, en especial Microsporum canis (Eurotiomycetes Onygenales, Arthrodermataceae), conocido colonizador de la piel y fanéras, causando infecciones superficiales en los animales que pueden transmitirse al hombre (especies zoofílicas). Estos felinos (aparentemente sanos), generalmente son 
sus portadores asintomáticos. Su ausencia en nuestra investigación ha sido reportada en trabajos similares y puede relacionarse al antagonismo de la variada y abundante micota fúngica queratinofílica encontrada en su pelaje (Moriello \& De Boer, 1991; Cabañes, 2000; Sierra et al. 2000; Levi et al., 2006).

Sólo encontramos 2 representantes de 2 familias de los Onygenales (Gymnoascaceae y Onygenaceae) (Currah, 1985), Gymnascella citrina (=Gymnoascus citrinus) y Chrysosporium keratinophilum. Varias especies de Gymnoascaceae han sido recuperadas desde el pelaje de animales y aves, tales como: Ctenomyces serratus, Gymnoascus reesii, G intermedius, y Gymnascella dankaliensis (=Gymnoascus dankaliensis) entre otros (Mantovani, 1978; Chabasse, 1988; Hubalek, 2000). Piontelli \& Toro (1987), detectan una especie cercana en la misma zona (G. dankaliensis) en el $(5.8 \%)$ y $(1.1 \%)$ en perro y gato respectivamente.

Los integrantes del género Chrysosporium son comunes en el pelaje de roedores y en aves domésticas y silvestres (Chabasse, 1988; Mancianti et al., 1993). Otros autores solo reportan el género como uno de los más comunes en gatos domésticos y felinos silvestres sin determinar la especie (Gambale et al. 1993; Guzmán-Chavez et al., 2000; Levi et al.,2006). En España, (Cabañes et al., 1996) lo aisló desde el (64.5\%) de los perros estudiados, describiendo una mayor frecuencia en verano que en otras estaciones. C. keratinophylum, tuvo la más alta frecuencia en gatos (25,3\%) en Valparaíso (Piontelli \& Toro,1987).

C. keratinophilum (teleomorfo Aphanoascus

keratinophilus), es una de las especies más frecuentes en el suelo y a pesar de ser cosmopolita y de amplia distribución parece ser insignificante en la patología humana y animal salvo algunas lesiones en uñas y piel (De Hoog et al., 2000).

En el estudio de Ali-Shayed et al.(1988), los hongos potencialmente patógenos oportunistas abarcaron casi el $90 \%$ de los hongos queratinofílicos recuperados desde el pelaje de gatos, concluyendo que este biotopo de los mamíferos domésticos es un excelente reservorio de una diversificada micota, situación casi idéntica a la ocurrida en la presente investigación, donde la frecuencia de los hongos potencialmente patógenos fue similar en porcentaje.

\section{CONCLUSIONES}

Se obtuvo un alto porcentaje de aislamientos de hongos queratinofílicos potencialmente patógenos de los géneros Aspergillus, Scopulariopsis, Fusarium y Alternaria. El género dominante y con mayor cantidad de especies (10) fue Aspergillus, con una mayor presencia en gatos de vida libre, siendo $\boldsymbol{A}$. flavus y $\boldsymbol{A}$. niger var. niger, las especies dominantes. Debido a su termotolerancia y capacidad de producir metabolitos tóxicos (micotoxinas), deben considerarse de importancia clínica en veterinaria, situación similar para la especie de mayor aislamiento como S.brevicaulis, por su capacidad infectiva queratinolítica y resistencia a casi todos los antimicóticos.

La mayoría de los hongos aislados fueron queratinofílicos $(98,11 \%)$. Los considerados queratinolíticos fueron solamente 2 especies: G. citrina y C. keratinophylum.

La ausencia de M.canis puede asociarse a las capacidades competitivas y antagónicas de las especies queratinofílicas dominantes. Esta situación poco común en estos felinos (aparentemente sanos), generalmente portadores asintomáticos, ha sido reportada anteriormente en la literatura.

De las variables en estudio, el sexo y tipo de pelo, no presentaron diferencia significativa para ninguna de las especies estudiadas, los gatos de vida libre presentaron mayor proporción de portadores de cepas queratinofilicas potencialmente patógenas, los gatos menores de un año presentaron mayor proporción de aislamientos de $\boldsymbol{S}$. brevicaulis, mientras los mayores de dos años mayor proporción de Aspergillus niger var. niger.

\section{REFERENCIAS}

Abbott, S.P.; Sigler, L. \& Currah, R.S. (1998). Microascus brevicaulis sp. nov., the teleomorph of Scopulariopsis brevicaulis, supports placement of Scopulariopsis with the Microascaceae. Mycologia 90:297-302

Aho, R. (1983). Saprophytic fungi isolated from the hair of domestic and laboratory animals with suspected dermatophytosis. Mycopathologia 83:65-73

Airaudi, D. \& Marchisio, F. V. (1996). Fungal biodiversity in the air of Turin. Mycopathologia 136:95-102

Ali-Shtayeh, M.S.; Arda, H-M.; Hassouna, M \& Shaheen, S.F.(1988). Keratinophilic fungi on the hair of cows, donkeys, rabbits, cats, and dogs from the West Bank of Jordan. Mycopathologia 104:109-121

Andreu, V.V. (2000). Detección de portadores de dermatofitos en conejos, hámsteres y ratones, no afectados clínicamente, mediante examen de cultivo micológico. Tesis para optar al título profesional de médico veterinario. Santiago, Chile, Univ. Santo Tomás, Escuela de Medicina Veterinaria. 74 p.

Bagy, M. (1986). Fungi on the hair of large mammals in Egypt. Mycopathologia 93:73-75

Boyanowski, K. J.; Ihrke, P. J.; Moriello, K. A.; Kass, P.H. (2000). Isolation of fungal flora from hair coats of shelter cats in the Pacific Coastal USA. Vet. Dermatol. 11:143-150

Cabañes, F.J.; Abarca, M.L.; Bragulat, M.R. \& Castellá, G. (1996). Seasonal Study of fungal biota of the fur of dogs. Mycopathologia 133:1-7 
Cabañes, F. J. (2000). Dermatofitosis animales recientes avances. Rev. Iberoam. Micol. 17:S8-S12

Camin, A.M.; Chabasse, D. \& Guiguen, C. (1998). Keratinophilic fungi associated with starlings (Sturnus vulgaris) in Brittany, France. Mycopathologia 143:9-14

Caretta, G.; Mancianti, F. \& Ajello, L. (1989). Dermmatophytes and keratinophilic fungi in cats and dogs. Mycoses $32: 620-626$

Cavalcanti, M.P.; Faustino, M.A.G.; Gomes-Filho, J.B.; Alves, L.C. (2003). Frequência de dermatófitos e fungos saprófitas em caninos e felinos com sintomatologia sugestiva de dermatopatia micótica atendidos no Hosp. Veter. da UFRPE. Clín. Vet., 24-28

Chabasse,D. (1988). Taxonomic study of keretinophilic fungi isolated from soil and mammals in France. Mycopathologia 101:133-140

Corfio, P, P. (1998). Diagnóstico diferencial de las dermatopatías infecciosas más frecuentes en caninos del Área Metropolitana determinado a través del Examen de Microscopía Directa. Tesis de grado para optar al título profesional de médico veterinario. Santiago, Chile, Univ. Santo Tomás, Esc. de Med. Veterinaria. 45p.

Cuenca-Estrella, M.; Gomez-Lopez, A.; Buitrago, M.J.; Mellado, E.; Garcia-Effron, G.; Rodriguez-Tudela, J.L.(2006) In Vitro Activities of 10 Combinations of Antifungal Agents against the Multiresistant Pathogen Scopulariopsis brevicaulis. Antimicrob. Agents Chemother. 50 (6):2248-2250

Currah,R.S.(1985).Taxonomy of the Onygenales: Arthrdermataceae, Gymnoascaceae, Myxotrichaceae and Onygenaceae. Mycotaxon, vol. 24:1-226

Day, M..Penhale WJ, Eger CE, Shaw SE, Kabay MJ, Robinson WF, Huxtable CR, Mills JN, Wyburn RS. (1986).Disseminated aspergillosis in dogs. Aust.Vet. J. 63:55-59

De Hoog, G.S.; Guarro, J.; Gene J. \& Figueras, M.J. (2000) Atlas of clinical Fungi. Sec. ed. Centraalbureau voor schimmelcultures, Utrecht, the Netherlands \& Universitat Rovira i Virgili, Reus España

Domsh, K.H.; Gams, W. \& Anderson, T. (1980). Compendium of soil fungi. Vol. I-II, Academic Press, London.

Elliot, G. S.; Whitney, M.S.; Reed, W. \& Tuite, S.F. (1984). Antemorte diagnosis of paecilomycosis in a cat. J Am Vet Med Ass.1:93-94

Filippello-Marchisio, V.; Fusconi, A. \&Queirio, F.L. (2000). Scopulariopsis brevicaulis: a keratinophilic or a keratinolytic fungus?. Mycoses 43:281-292

Flores,C.; Gutierrez, E \& Biefang, F. (1954). Paralelismo etiológico entre la tinea del perro ydel gato y la del niño en la ciudad de Santiago. Rev.Soc. Med. Vet. de Chile 13:207-211

Gambale, W.; Larsson, C.E.; Moritami, M.M.; Corrêa, B.; Paula, C.R.; Framil, V.M.S.(1993). Dermatophytes and other fungi of the haircoat of cats without dermatophyosis in the city of São Paulo, Brasil. Fel. Pract., 21:29-33

González,C.J. (1986). Micosis cutáneas y subcutáneas producidas por hongos oportnistas enimales de compañía. Rev, Iber. Micol. 3:
$119-129$

Greene, C. (1998). Infectious Disease of the Dog and Cat. W.B. Saunders Co. Philadelphia, PA;

Gugnani, H.C.(2000). Nondermatophytic filamentous keratinophilic fungi and their role in human infection. In: biology of Dermatophytes and other keratinophilic fungi. Kushwaha, R.K.S. \& Guarro, J. (eds.) pp.109-114.

Guzmán-Chavez, R.; Segundo-Zaragoza,C. \& Cervantes, R.A. (2000).Presence of keratinophilic fungi with special reference to dermatophytes on the haircoat of dogs and cats in a Mexico and Nezahualcoyotl cities. Rev. Latinoam. Microbiol. 42:41-44

Hubalek, Z. (2000). Keratinophilic fungi associated with free living mammals and birds. Rev. Iberoam. Micol. 17:93-103

Jiménez F, M.. (1980). Contribución al estudio de las dermatomicosis en especies menores. Tesis para optar al título de médico veterinerio y licenciado en ciencias pecuarias y médico veterinarias. Santiago, Chile, Universidad de Chile. Facultad de Ciencias Veterinarias. $36 \mathrm{p}$.

Klich, M.A. (2002). Identification of common Aspergillus species. CBS. Utrecht, The Netherlands.

Kluger, E. K.; Della Torre, P. K.; Martin, P.; Krockenberger, M. B.; Malik, R. (2004). Concurrent Fusarium chlamydosporum and Microsphaeropsis arundinis infections in a cat. J. Feline Med. Surg. 6:271-7

Khosravi, A.R.(1996). Fungal flora of the hair coat of stray cats in Iran. Mycoses 39:241-243

Kunert, J. (2000) Physiology of keratinophilic fungi. In: biology of Dermatophytes and other keratinophilic fungi. Kushwaha, R.K.S. \& Guarro, J. (eds.) pp.77-85

Leslie, J.F. \& Summerell, B.A. (2005). The Fusarium laboratory Manual. Blackwell Publishing. Iowa USA.

Levi, H.B.; Luzes, J.D.; Ramiro, S.H.; Friciello, R.H.; Acqua, S.D. (2006). Isolation of Microsporum canis from the haircoat of health wild felids kept in capttivity in Brazil. Braz. J. Microbiol 37: $148-152$

Mancianti, F.;Papini, R. \& Poli, A.(1993).Mycological survey from coats of red foxes in Italy. J. Mycol Med. 3:109-110

Mantovani, A. (1978). The role of animals in the epidemiology of the mycoses. Mycopathologia. 65:61-66

Mariat, F. \& Adam-Campos, C. (1967). La technique du carré du tapis, méthode simple de prévelement dans le mycoses superficielles. Ann. Inst. Pasteur. 113:666-668

Migrino, R. Q.; Hall,G.S. \& Longworth, D.L. (1995). Deep tissue infections caused by Scopulariopsis brevicaulis: report of a case of prosthetic valve endocarditis and review. Clin. Infect. Dis. $21: 672-674$

Moriello, K.A. \& De Boer, D.J.(1991). Fungal flora of the haircoat of cat with an without dematophytosis. J. Med. Vet. Mycol. 29:285-292

Mortellaro, C.M.; Franca, P.D. \& Caretta, G. (1989). Aspergillus 
fumigatus, the causative agent of infection of the frontal sinuses and nasal chambers of the dog. Mycoses 32:327-35

Mullanei, T.; Levin, S. \& Indrieri, R.J. (1983).Disseminated aspergillosis in a dog. J. Amer. Vet. Med. Associa. 182:516-518

Paixao, G..C.; Sidrim, J.J.C.; Campos, G.M.M.; Brilhante, R.S.N.; Rocha, M.F.G.. (2001). Dermatophytes and saprobe fungi isolated from dogs and cats in the city of Fortaleza, Brazil. Arq. Bras. Med. Vet. Zootec. 53:568-573

Pérez, G. E, (2005). Estudio de reservorios fungicos de dermatófitos a partir de fomites provenientes de hospitales veterinarios. Tesis para optar al título profesional de médico veterinario. Universidad Santo Tomás, Escuela de Medicina Veterinaria. 61p.

Piontelli, E. \& Toro, M.A. (1987). Los animales domésticos (perros y gatos) como reservorio fúngico. Boletín Micológico 4:149-158

Reyes, J. E.C. (1998). Determinación de portadores asintomáticos de dermatofitos en caninos del área Metropolitana. Tesis para optar al título profesional de médico veterinario. Santiago, Chile, Univ. Santo Tomás, Escuela de Médicina Veterinaria. pp.30-33.

Romano, C.; Papini, M.; Ghilardi, A. \& Gianni, C. (2005). Onychomycosis in children: a survey of 46 cases. Mycoses 48:430437

Rotstein, D. S.; Thomas, R.; Heimick, K.; Citino, S.B.; Taylor, S.K.; Dumbar, M.R.(1999). Dermatophyte infection in free-ranging Florida panthers (Felix concolor coryi). J. Zoo Wildl. Med. 30:281-284

Samson, R.A., Hoekstra, E.S.: Frisvald, J.C. \& Filtenborg, O. (2000). Introduction to food-and airborne fungi. CBS. Utrecht, The Netherlands.
San Juan, R.; Berenguer, J. \& Aguado,J.M. (2004). Hongos filamentosos emergentes: Scedosporium. Contro Calidad SEIMC. $1-6$

Scott, D.W.; Miller, W.H. \& Griffin, C,E. (eds.)(2001). Small animal dermatology. Sexta Edición. Editorial W. B. Saunders Company. EUA. pp. 330-415

Sharma, R. \& Rajak, R. (2003) Keratinophilic Fungi: Nature's keratin degrading machines. Their isolation, identification and ecologycal rol. Resonance September:28-40

Schinabeck, M. K. \& Ghannoum, M.A. (2003). Human hyalohyphomycoses: a review of human infections due to Acremonium spp., Paecilomyces spp., Penicillium spp., and Scopulariopsis spp. J. Chemother. 15:5-15

Sierra, P.; Guillot, J.; Jacob, H.; Bussiéras, S.; Chermette, R. (2000). Fungal flora on cutaneous and mucosal surfaces of cats infected with feline immunodeficiency virus or feline leukemia virus. Am. J. Vet. Res. 61:158-161

Sparkes, A.H.; Gruffydd-Jones, T.J.; Shaw, S.E.; Wright, A.L.; Stokes, C.R. (1993). Epidemiological and diagnostic features of canine and feline dermatophytosis in the United Kingdom from 1956 to 1991. Vet, Rec. 133:57-61

Thomson, M. P. (2002). Prevalencia de dermatofitosis y estado de portador de dermatofitos en caninos del área Sur de la región Metropolitana. Tesis para optar al título profesional de médico veterinario. Santiago, Chile, Universidad Santo Tomás, Escuela de Medicina Veterinaria.65p.

Zaror, L.; Fischman, O.; Vilanova, A. \& Levites, J. (1986). The role of cats and $\operatorname{dog} s$ in the epidemiological cycle of Microsporum canis. Mykosen 29:185-188 